We identified 179 infants with a diagnosis of pertussis at discharge and enrolled 97 (54\%). These did not differ from the non-enrolled infants in age, sex, ethnicity, gestation, birth weight, or social deprivation score. To ensure that the controls were a representative sample of children with non-pertussis coughing illnesses we compared the 98 enrolled control infants with two other groups of infants admitted to hospital with such illnesses: 227 infants with cough who had not been approached for enrolment and 78 infants with cough who had been identified as eligible but for whom informed consent was not obtained. The controls and the other two groups did not differ in age, proportion of infants of non-European ethnicity, or social deprivation index.

We obtained nasopharyngeal samples for culture from 95 (98\%) of the 97 infants with pertussis and for polymerase chain reaction from $83(86 \%)$. We identified $B$ pertussis by culture in $32(34 \%)$, by polymerase chain reaction in $73(75 \%)$, and by either method in $76(80 \%)$ infants.

The infants with pertussis were younger than the controls and more likely to have mothers with only primary school education (odds ratio $11.78,95 \%$ confidence interval 2.02 to 225.37) and to live in more crowded households (2.12, 1.17 to 3.89 ) and households in the most socially deprived fifth $(2.21,1.18$ to 4.23). We found no differences between the two groups in other characteristics of the infant (gestation, birth weight, ethnicity, or breast feeding), mother (age, marital status, and smoking), or household (mobility, smokers, occupation, and socioeconomic status).

The table shows associations between delayed immunisations and risk of admission to hospital with pertussis. In the multivariate analysis we found an increased risk associated with delay in the first, second, or third immunisation or any combination of these (odds ratio 4.50). Analysis by individual dose of vaccine showed that an increased risk of pertussis was associated with delay in the third dose (odds ratio 6.09). Including all variables describing infant, maternal, and household characteristics in the model did not alter the importance of increased risk associated with any delayed immunisations (odds ratio 6.13, 1.13 to 47.07).

\section{Comment}

Delayed immunisation is a specific risk factor for admission to hospital with pertussis rather than being a marker of infants at increased risk of admission to hospital for any acute respiratory illness. Improving on-time delivery of immunisations can be expected to decrease the admission rate for pertussis in New Zealand.

Contributors: CCG enrolled participants, analysed the data, and drafted the paper. RS directed study design and data analysis. DL initiated and designed the project. RF enrolled cases and assisted with the ethical application. JS advised on study design, data management, and analysis. RM designed and performed all of the polymerase chain reaction assays. DK identified cases and controls. MR enrolled cases and controls and supervised other interviewers. All authors revised the paper, CCG wrote the paper and is the guarantor.

Funding: This research was supported by grants from the National Child Health Research Foundation and the Health Research Council of New Zealand.

Competing interests: None declared.

The regional ethics committee approved the study.

1 Pollock TM, Miller E, Lobb J. Severity of whooping cough in England before and after the decline in pertussis immunisation. Arch Dis Child 1984;59:162-5.

2 Guris D, Strebel PM, Bardenheier B, Brennan M, Tachdjian R, Finch E, Wharton M, et al. Changing epidemiology of pertussis in the United States: increasing reported incidence among adolescents and adults, 1990-1996. Clin Infect Dis 1999;28:1230-7.

Reid S, Lennon D, Thomas M, O'Connor P, Baker M, Mansoor O. Pertussis control in New Zealand. N Z Med J 1994; 107:460-2, 463-6.

4 Dietz VJ, Zell ER, Stevenson J. Defining delayed immunization. Pediatr Infect Dis J 1993;12:353-4.

Crampton P, Davis P. Measuring deprivation and socioeconomic status: why and how? N Z Public Health Rep 1998;5:81-4.

(Accepted 11 November 2002)

\title{
Science commentary: Pertussis immunisation
}

Tolerance for pertussis (whooping cough) immunisation is highest when given early, and on time. Since the disease is most severe in young infants, early completion of immunisation is critical. Pertussis immunisation in the United Kingdom is presently given at 2, 3, and 4 months. This "accelerated" course was introduced in 1990, and there is good direct evidence that the early schedule is tolerated better than the previous (later) schedule. ${ }^{1}$ This negates the idea that parents sometimes have that babies "cope" better with vaccination when they are older and bigger. Efficacy of the pertussis vaccine used in England and Wales is high although it wanes with increasing age. ${ }^{2}$

If primary immunisation is delayed or not completed, child health will be compromised. While typhoid, diphtheria, and tetanus are not endemic in the United Kingdom, pertussis is present and does cause morbidity and mortality. Pertussis causes most morbidity before the age of 8 weeks (before immunisation begins). The number of hospital admissions for pertussis in all ages was 853 in 1999 (E Miller, personal communication, 2001) and almost half of these were in children under 3 months of age. Without the accelerated course, these rates would be higher, and overall protection depends on the course being completed.

The youngest babies are probably getting pertussis from older children, who usually suffer only a mild illness, and for this reason preschool boosters for pertussis were introduced from October 2001. One study that looked at whole cell pertussis preschool boosters found that while antibody responses were high, the rate of reactions increased, ${ }^{3}$ whereas another study found that acellular pertussis boosters result in good immunogenicity and do not produce more reactions. ${ }^{4}$

1 Miller E, Ashworth LAE, Redhead K, Thornton C, Waight PA, Coleman T Effect of schedule on reactogenicity and antibody persistence of acellular and whole-cell pertussis vaccines: value of laboratory tests as predictors of clinical performance. Vaccine 1997;15:51-60.

2 Van Buynder PG, Owen D, Vurdien JE, Andrews NJ, Matthews RC, Miller E. Bordetella pertussis surveillance in England and Wales: 1995-7. Epidemiol Infect 1999;123:403-11.

3 Miller E, Rush M, Ashworth LAE, Coleman TJ, Rossini J, Ahmed OA, et al. Antibody responses and reactions to the whole cell pertussis component of a combined diphtheria/tetanus/pertussis vaccine given at school entry. Vaccine 1995;13:1183-6.

4 Miller E, Waight P, Laurichesse H, Andrews N, Thornton C, Sesardic D, et al. Immunogenicity and reactogenicity of acellular diphtheria/tetanus/ pertussis vaccines given as a pre-school booster: effect of simultaneous pertussis vaccines given as a pre-school booster:
administration of MMR. Vaccine 2001;19:3904-11.
Community

Paediatrics,

Canterbury District

Health Board,

Private Bag 4345,

Christchurch, New

Zealand

Rodney Ford

clinical associate

professor

Virology and

Immunology,

Auckland District

Health Board, New

Zealand

Rosalie Menzies

scientific officer

Correspondence to: C C Grant

cc.grant@auckland.

ac.nz 